\title{
On a Parametric Mulholland-Type Inequality and Applications
}

\author{
Bicheng Yang, ${ }^{1}$ Meifa Huang, ${ }^{2}$ and Yanru Zhong $\mathbb{D}^{3}$ \\ ${ }^{1}$ Department of Mathematics, Guangdong University of Education, Guangzhou, Guangdong 510303, China \\ ${ }^{2}$ School of Mechanical Science and Electrical Engineering, Guilin University of Electronic Technology, Guilin, Guangxi 541004, China \\ ${ }^{3}$ School of Computer Science and Information Security, Guilin University of Electronic Technology, Guilin, Guangxi 541004, China
}

Correspondence should be addressed to Yanru Zhong; 18577399236@163.com

Received 29 March 2019; Accepted 9 May 2019; Published 20 May 2019

Academic Editor: Wing-Sum Cheung

Copyright (C) 2019 Bicheng Yang et al. This is an open access article distributed under the Creative Commons Attribution License, which permits unrestricted use, distribution, and reproduction in any medium, provided the original work is properly cited.

In this paper, by the use of the weight functions, and the idea of introducing parameters, a discrete Mulholland-type inequality with the general homogeneous kernel and the equivalent form are given. The equivalent statements of the best possible constant factor related to a few parameters are provided. As applications, the operator expressions and a few particular examples are considered.

\section{Introduction}

Assuming that $0<\sum_{m=1}^{\infty} a_{m}^{2}<\infty$ and $0<\sum_{n=1}^{\infty} b_{n}^{2}<\infty$, we have the following discrete Hilbert's inequality with the best possible constant factor $\pi$ (cf. [1], Theorem 315):

$$
\sum_{m=1}^{\infty} \sum_{n=1}^{\infty} \frac{a_{m} b_{n}}{m+n}<\pi\left(\sum_{m=1}^{\infty} a_{m}^{2} \sum_{n=1}^{\infty} b_{n}^{2}\right)^{1 / 2}
$$

We still have the following Mulholland's inequality with the same best possible constant $\pi$ (cf. [1], Theorem 343):

$$
\sum_{m=2 n=2}^{\infty} \sum_{n}^{\infty} \frac{a_{m} b_{n}}{\ln m n}<\pi\left(\sum_{m=2}^{\infty} m a_{m}^{2} \sum_{n=2}^{\infty} n b_{n}^{2}\right)^{1 / 2} .
$$

If $0<\int_{0}^{\infty} f^{2}(x) d x<\infty$ and $0<\int_{0}^{\infty} g^{2}(y) d y<\infty$, then we have the following Hilbert's integral inequality:

$$
\begin{aligned}
& \int_{0}^{\infty} \int_{0}^{\infty} \frac{f(x) g(y)}{x+y} d x d y \\
& \quad<\pi\left(\int_{0}^{\infty} f^{2}(x) d x \int_{0}^{\infty} g^{2}(y) d y\right)^{1 / 2}
\end{aligned}
$$

with the best possible constant factor $\pi$ (cf. [1], Theorem 316).
Inequalities (1), (2), and (3) and their extensions with the conjugate exponents $(p, q)(p>1,1 / p+1 / q=1)$ and independent parameters are important in analysis and its applications (cf. [2-13]).

The following half-discrete Hilbert-type inequality was provided (cf. [1], Theorem 351). If $K(x)(x>0)$ is decreasing, $p>1,1 / p+1 / q=1,0<\phi(s)=\int_{0}^{\infty} K(x) x^{s-1} d x<\infty$, then

$$
\int_{0}^{\infty} x^{p-2}\left(\sum_{n=1}^{\infty} K(n x) a_{n}\right)^{p} d x<\phi^{p}\left(\frac{1}{q}\right) \sum_{n=1}^{\infty} a_{n}^{p} .
$$

Some new extensions of (4) were provided by [14-19].

In 2016, by the use of the technique of real analysis, Hong [20] considered some equivalent statements of the extensions of (1) with the best possible constant factor related to a few parameters. The other similar works about the extensions of (3) were provided by [21-25].

In this paper, according to the way given by [20], by the use of the weight functions and the idea of introducing parameters, a discrete Mulholland-type inequality with the general homogeneous kernel and the equivalent form are given, which is an extension of (2). The equivalent statements of the best possible constant factor related to a few parameters are provided. As applications, the operator expressions and a few particular examples are considered. 


\section{Some Lemmas}

In what follows, we suppose that $p>1,1 / p+1 / q=1, \lambda \in$ $\mathrm{R}, \lambda_{i}, \lambda-\lambda_{i} \leq 1(i=1,2), k_{\lambda}(x, y)$ is a positive homogeneous function of degree $-\lambda$, satisfying, for any $u, x, y>0$,

$$
k_{\lambda}(u x, u y)=u^{-\lambda} k_{\lambda}(x, y) .
$$

Also, $k_{\lambda}(x, y)$ is decreasing with respect to $x, y>0$ (or $\left.(\partial / \partial x) k_{\lambda}(x, y) \leq 0,(\partial / \partial y) k_{\lambda}(x, y) \leq 0(x, y>0)\right)$, such that, for $\gamma=\lambda_{1}, \lambda-\lambda_{2}$,

$$
k_{\lambda}(\gamma):=\int_{0}^{\infty} k_{\lambda}(u, 1) u^{\gamma-1} d u \in \mathrm{R}_{+}=(0, \infty) .
$$

We still assume that $a_{m}, b_{n} \geq 0(m, n \in \mathrm{N} \backslash\{1\}=\{2,3, \ldots\})$, satisfying

$$
\begin{aligned}
& 0<\sum_{m=2}^{\infty} \frac{\ln ^{p\left[1-\left(\left(\lambda-\lambda_{2}\right) / p+\lambda_{1} / q\right)\right]-1} m}{m^{1-p}} a_{m}^{p}<\infty \\
& \text { and } 0<\sum_{n=2}^{\infty} \frac{\ln ^{q\left[1-\left(\lambda_{2} / p+\left(\lambda-\lambda_{1}\right) / q\right)\right]-1} n}{n^{1-p}} b_{n}^{q}<\infty .
\end{aligned}
$$

Definition 1. Define the following weight functions:

$$
\begin{aligned}
\omega_{\lambda}\left(\lambda_{2}, m\right):=\ln ^{\lambda-\lambda_{2}} m \sum_{n=2}^{\infty} k_{\lambda}(\ln m, \ln n) & \frac{\ln ^{\lambda_{2}-1} n}{n} \\
& (m \in \mathrm{N} \backslash\{1\}), \\
\omega_{\lambda}\left(\lambda_{1}, n\right):=\ln { }^{\lambda-\lambda_{1}} n \sum_{m=2}^{\infty} k_{\lambda}(\ln m, \ln n) & \frac{\ln ^{\lambda_{1}-1} m}{m} \\
& (n \in \mathrm{N} \backslash\{1\}) .
\end{aligned}
$$

Lemma 2. We have the following inequalities:

$$
\begin{aligned}
& \omega_{\lambda}\left(\lambda_{2}, m\right)<k_{\lambda}\left(\lambda-\lambda_{2}\right) \quad(m \in \mathrm{N} \backslash\{1\}), \\
& \omega_{\lambda}\left(\lambda_{1}, n\right)<k_{\lambda}\left(\lambda_{1}\right) \quad(n \in \mathrm{N} \backslash\{1\}) .
\end{aligned}
$$

Proof. For $\lambda_{2}-1 \leq 0$, it is evident that $k_{\lambda}(\ln m, \ln t)\left(\ln ^{\lambda_{2}-1} t\right) / t$ is a strictly decreasing function with respect to $t>1$. By the decreasing property, setting $u=\ln m / \ln t$, it follows that

$$
\begin{aligned}
\omega_{\lambda}\left(\lambda_{2}, m\right) & <\ln ^{\lambda-\lambda_{2}} m \int_{1}^{\infty} k_{\lambda}(\ln m, \ln t) \frac{\ln ^{\lambda_{2}-1} t}{t} d t \\
& =\int_{0}^{\infty} k_{\lambda}(u, 1) u^{\left(\lambda-\lambda_{2}\right)-1} d u=k_{\lambda}\left(\lambda-\lambda_{2}\right) .
\end{aligned}
$$

Hence, we have (10). For $\lambda_{1}-1 \leq 0$, it is evident that $k_{\lambda}(\ln t, \ln n)\left(\ln ^{\lambda_{1}-1} t\right) / t$ is a strictly decreasing function with respect to $t>1$. By the decreasing property, setting $u=$ $\ln t / \ln n$, we find that

$$
\begin{aligned}
\omega_{\lambda}\left(\lambda_{1}, n\right) & <\ln ^{\lambda-\lambda_{1}} n \int_{1}^{\infty} k_{\lambda}(\ln t, \ln n) \frac{\ln ^{\lambda_{1}-1} t}{t} d t \\
& =\int_{0}^{\infty} k_{\lambda}(u, 1) u^{\lambda_{1}-1} d u=k_{\lambda}\left(\lambda_{1}\right) .
\end{aligned}
$$

Hence, we have (11).
Lemma 3. We have the following inequality:

$$
\begin{aligned}
I:= & \sum_{n=2}^{\infty} \sum_{m=2}^{\infty} k_{\lambda}(\ln m, \ln n) a_{m} b_{n}<k_{\lambda}^{1 / p}\left(\lambda-\lambda_{2}\right) \\
& \cdot k_{\lambda}^{1 / q}\left(\lambda_{1}\right)\left\{\sum_{m=2}^{\infty} \frac{\ln ^{p\left[1-\left(\left(\lambda-\lambda_{2}\right) / p+\lambda_{1} / q\right)\right]-1} m}{m^{1-p}} a_{m}^{p}\right\}^{1 / p} \\
& \cdot\left\{\sum_{n=2}^{\infty} \frac{\ln ^{q\left[1-\left(\left(\lambda-\lambda_{1}\right) / q+\lambda_{2} / p\right)\right]-1} n}{n^{1-q}} b_{n}^{q}\right\}^{1 / q} .
\end{aligned}
$$

Proof. By Hölder's inequality with weight (cf. [26]), we obtain

$$
\begin{aligned}
I: & =\sum_{n=2}^{\infty} \sum_{m=2}^{\infty} k_{\lambda}(\ln m, \ln n)\left[\frac{\ln { }^{\left(\lambda_{2}-1\right) p} n}{n^{1 / p}} \frac{\ln ^{\left(1-\lambda_{1}\right) / q} m}{m^{-1 / q}} a_{m}\right] \\
& \times\left[\frac{\ln { }^{\left(\lambda_{1}-1\right) / q} m}{\mathrm{~m}^{1 / q}} \frac{\ln ^{\left(1-\lambda_{2}\right) / p} n}{n^{-1 / p}} b_{n}\right] \\
& \leq\left[\sum_{m=2}^{\infty} \sum_{n=2}^{\infty} k_{\lambda}(\ln m, \ln n) \frac{\ln ^{\lambda_{2}-1} n}{n} \frac{\ln ^{(p-1)\left(1-\lambda_{1}\right)} m}{m^{1-p}}\right. \\
& \left.\cdot a_{m}^{p}\right]^{1 / p} \times\left[\sum_{n=2}^{\infty} \sum_{m=2}^{\infty} k_{\lambda}(\ln m, \ln n) \frac{\ln ^{\lambda_{1}-1} m}{m^{2}}\right. \\
& . \frac{\ln ^{(q-1)\left(1-\lambda_{2}\right)-1} n b^{1 / q}}{n^{1-q}}=\left\{\sum_{m=2}^{\infty} \omega_{\lambda}\left(\lambda_{2}, m\right)\right. \\
& \left.. \frac{\ln ^{p\left[1-\left(\left(\lambda-\lambda_{2}\right) / p+\lambda_{1} / q\right)\right]-1} m}{m^{1-p}} a_{m}^{p}\right\}^{1 / p} \times\left\{\sum_{n=2}^{\infty} \omega_{\lambda}\left(\lambda_{1}, n\right)\right. \\
& \left.\cdot \frac{\ln ^{q\left[1-\left(\left(\lambda-\lambda_{1}\right) / q+\lambda_{2} / p\right)\right]-1} n}{n^{1-q}} b_{n}^{q}\right\}^{1 / q} \cdot
\end{aligned}
$$

Then by (10) and (11), we have (14).

Remark 4. By (14), for $\lambda_{1}+\lambda_{2}=\lambda$, we find

$$
\begin{array}{r}
0<\sum_{m=2}^{\infty} \frac{\ln ^{p\left(1-\lambda_{1}\right)-1} m}{m^{1-p}} a_{m}^{p}<\infty \\
\text { and } 0<\sum_{n=2}^{\infty} \frac{\ln ^{q\left(1-\lambda_{2}\right)-1} n}{n^{1-p}} b_{n}^{q}<\infty,
\end{array}
$$

and the following inequality:

$$
\begin{aligned}
& \sum_{n=2}^{\infty} \sum_{m=2}^{\infty} k_{\lambda}(\ln m, \ln n) a_{m} b_{n}<k_{\lambda}\left(\lambda_{1}\right) \\
& \cdot\left[\sum_{m=2}^{\infty} \frac{\ln ^{p\left(1-\lambda_{1}\right)-1} m}{m^{1-p}} a_{m}^{p}\right]^{1 / p}\left[\sum_{n=2}^{\infty} \frac{\ln ^{q\left(1-\lambda_{2}\right)-1} n}{n^{1-q}} b_{n}^{q}\right]^{1 / q} .
\end{aligned}
$$


In particular, for $p=q=2$, we have

$$
\begin{aligned}
& \sum_{n=2}^{\infty} \sum_{m=2}^{\infty} k_{\lambda}(\ln m, \ln n) a_{m} b_{n} \\
& \quad<k_{\lambda}\left(\lambda_{1}\right)\left(\sum_{m=2}^{\infty} \frac{m}{\ln ^{2 \lambda_{1}-1} m} a_{m}^{2} \sum_{n=2}^{\infty} \frac{n}{\ln ^{2 \lambda_{2}-1} n} b_{n}^{2}\right)^{1 / 2} .
\end{aligned}
$$

For $\lambda=1, k_{1}(x, y)=1 /(x+y), \lambda_{1}=\lambda_{2}=1 / 2$, (18) reduces to (2). Hence, (17) is an extension of (18) and (2).

Lemma 5. The constant factor $k\left(\lambda_{1}\right)$ in (17) is the best possible.

Proof. For any $\varepsilon>0$, we set

$$
\begin{aligned}
& \tilde{a}_{m}:=\frac{\ln ^{\lambda_{1}-\varepsilon / p-1} m}{m}, \\
& \tilde{b}_{n}:=\frac{\ln ^{\lambda_{2}-\varepsilon / q-1} n}{n} \\
& (m, n \in \mathrm{N} \backslash\{1\}) .
\end{aligned}
$$

If there exists a constant $M\left(M \leq k_{\lambda}\left(\lambda_{1}\right)\right)$, such that (17) is valid when replacing $k_{\lambda}\left(\lambda_{1}\right)$ by $M$, then, in particular, we have

$$
\begin{aligned}
\widetilde{I}:= & \sum_{n=2}^{\infty} \sum_{m=2}^{\infty} k_{\lambda}(\ln m, \ln n) \tilde{a}_{m} \widetilde{b}_{n} \\
& <M\left[\sum_{m=2}^{\infty} \frac{\ln ^{p\left(1-\lambda_{1}\right)-1} m}{m^{1-p}} \widetilde{a}_{m}^{p}\right]^{1 / p} \\
& \cdot\left[\sum_{n=2}^{\infty} \frac{\ln ^{q\left(1-\lambda_{2}\right)-1} n^{q}}{n^{1-p}} \widetilde{b}_{n}^{1 / q} .\right.
\end{aligned}
$$

We obtain

$$
\begin{aligned}
\widetilde{I} & <M\left[\sum_{m=2}^{\infty} \frac{\ln ^{p\left(1-\lambda_{1}\right)-1} m}{m^{1-p}} \frac{\ln ^{p \lambda_{1}-\varepsilon-p} m}{m^{p}}\right]^{1 / p} \\
& \cdot\left[\sum_{n=2}^{\infty} \frac{\ln ^{q\left(1-\lambda_{2}\right)-1} n}{n^{1-q}} \frac{\ln ^{q \lambda_{2}-\varepsilon-1} n}{n^{q}}\right]^{1 / q} \\
& =M\left(\frac{\ln ^{-\varepsilon-1} 2}{2}+\sum_{m=3}^{\infty} \frac{\ln ^{-\varepsilon-1} m}{m}\right) \\
& <M\left(\frac{\ln ^{-\varepsilon-1} 2}{2}+\int_{2}^{\infty} \frac{\ln ^{-\varepsilon-1} t}{t} d t\right) \\
& =\frac{M}{\varepsilon \ln ^{\varepsilon} 2}\left(\frac{\varepsilon}{2 \ln 2}+1\right) .
\end{aligned}
$$

By the decreasing property and Fubini theorem (cf. [27]), we find

$$
\begin{aligned}
& \widetilde{I}=\sum_{n=2}^{\infty} \sum_{m=2}^{\infty} k_{\lambda}(\ln m, \ln n) \frac{\ln ^{\lambda_{1}-1} m}{m \ln ^{\varepsilon / p} m} \cdot \frac{\ln ^{\lambda_{2}-1} n}{n \ln ^{\varepsilon / q} n} \\
& \geq \int_{2}^{\infty}\left(\int_{2}^{\infty} k_{\lambda}(\ln x, \ln y) \frac{\ln ^{\lambda_{1}-\varepsilon / p-1} x}{x}\right. \\
& \left.\cdot \frac{\ln ^{\lambda_{2}-\varepsilon / q-1} y}{y} d x\right) d y\left(u=\frac{\ln x}{\ln y}\right) \\
& =\int_{2}^{\infty} \frac{\ln ^{-\varepsilon-1} y}{y}\left(\int_{\ln 2 / \ln y}^{\infty} k_{\lambda}(u, 1)\right. \\
& \left.\cdot u^{\lambda_{1}-\varepsilon / p-1} d u\right) d y \\
& =\int_{2}^{\infty} \frac{\ln ^{-\varepsilon-1} y}{y}\left(\int_{\ln 2 / \ln y}^{1} k_{\lambda}(u, 1)\right. \\
& \left.\cdot u^{\lambda_{1}-\varepsilon / p-1} d u\right) d y \\
& +\int_{2}^{\infty} \frac{\ln ^{-\varepsilon-1} y}{y}\left(\int_{1}^{\infty} k_{\lambda}(u, 1) u^{\lambda_{1}-\varepsilon / p-1} d u\right) d y \\
& =\int_{0}^{1}\left(\int_{\eta+2^{1 / u}}^{\infty} \frac{\ln ^{-\varepsilon-1} y}{y} d y\right) k_{\lambda}(u, 1) u^{\lambda_{1}-\varepsilon / p-1} d u \\
& +\frac{1}{\varepsilon \ln ^{\varepsilon} 2} \int_{1}^{\infty} k_{\lambda}(u, 1) u^{\lambda_{1}-\varepsilon / p-1} d u \\
& =\frac{1}{\varepsilon \ln ^{\varepsilon} 2}\left(\int_{0}^{1} k_{\lambda}(u, 1) u^{\lambda_{1}+\varepsilon / q-1} d u+\int_{1}^{\infty} k_{\lambda}(u, 1)\right. \\
& \left.\cdot u^{\lambda_{1}-\varepsilon / p-1} d u\right) \cdot
\end{aligned}
$$

Then we have

$$
\begin{aligned}
& \int_{0}^{1} k_{\lambda}(u, 1) u^{\lambda_{1}+\varepsilon / q-1} d u+\int_{1}^{\infty} k_{\lambda}(u, 1) u^{\lambda_{1}-\varepsilon / p-1} d u \\
& \quad<M\left(\frac{\varepsilon}{2 \ln 2}+1\right) .
\end{aligned}
$$

For $\varepsilon \longrightarrow 0^{+}$, by Fatou lemma (cf. [27]), we find

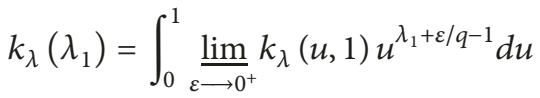

$$
\begin{aligned}
& \left.+\int_{1}^{\infty} \underset{\varepsilon \longrightarrow 0^{+}}{\varliminf_{\lambda}} k_{\lambda}(u, 1) u^{\lambda_{1}-\varepsilon / p-1} d u\right] \\
& \leq \varliminf_{\varepsilon \longrightarrow 0^{+}}\left(\int_{0}^{1} k_{\lambda}(u, 1) u^{\lambda_{1}+\varepsilon / q-1} d u\right. \\
& \left.+\int_{1}^{\infty} k_{\lambda}(u, 1) u^{\lambda_{1}-\varepsilon / p-1} d u\right) \leq M .
\end{aligned}
$$

Hence, $M=k_{\lambda}\left(\lambda_{1}\right)$ is the best possible constant factor of (17). 
Remark 6. Setting $\hat{\lambda}_{1}:=\left(\lambda-\lambda_{2}\right) / p+\lambda_{1} / q, \hat{\lambda}_{2}:=\left(\lambda-\lambda_{1}\right) / q+$ $\lambda_{2} / p$, we find

$$
\begin{aligned}
\hat{\lambda}_{1}+\widehat{\lambda}_{2} & =\frac{\lambda-\lambda_{2}}{p}+\frac{\lambda_{1}}{q}+\frac{\lambda-\lambda_{1}}{q}+\frac{\lambda_{2}}{p}=\frac{\lambda}{p}+\frac{\lambda}{q}=\lambda, \\
\hat{\lambda}_{1} & \leq \frac{1}{p}+\frac{1}{q}=1, \\
\hat{\lambda}_{2} & \leq \frac{1}{q}+\frac{1}{p}=1,
\end{aligned}
$$

and by Hölder's inequality (cf. [26]), we have

$$
\begin{aligned}
0< & k_{\lambda}\left(\lambda-\widehat{\lambda}_{2}\right)=k_{\lambda}\left(\widehat{\lambda}_{1}\right)=k_{\lambda}\left(\frac{\lambda-\lambda_{2}}{p}+\frac{\lambda_{1}}{q}\right) \\
& =\int_{0}^{\infty} k_{\lambda}(u, 1) u^{\left(\lambda-\lambda_{2}\right) / p+\lambda_{1} / q-1} d u \\
& =\int_{0}^{\infty} k_{\lambda}(u, 1)\left(u^{\left(\lambda-\lambda_{2}-1\right) / p}\right)\left(u^{\left(\lambda_{1}-1\right) / q}\right) d u \\
& \leq\left(\int_{0}^{\infty} k_{\lambda}(u, 1) u^{\lambda-\lambda_{2}-1} d u\right)^{1 / p} \\
& \cdot\left(\int_{0}^{\infty} k_{\lambda}(u, 1) u^{\lambda_{1}-1} d u\right)^{1 / q}=k_{\lambda}^{1 / p}\left(\lambda-\lambda_{2}\right) \\
& \cdot k_{\lambda}^{1 / q}\left(\lambda_{1}\right)<\infty .
\end{aligned}
$$

We can rewrite (14) as follows:

$$
\begin{aligned}
I< & k_{\lambda}^{1 / p}\left(\lambda-\lambda_{2}\right) k_{\lambda}^{1 / q}\left(\lambda_{1}\right)\left[\sum_{m=2}^{\infty} \frac{\ln ^{p\left(1-\hat{\lambda}_{1}\right)-1} m}{m^{1-p}} a_{m}^{p}\right]^{1 / p} \\
& \cdot\left[\sum_{n=2}^{\infty} \frac{\ln ^{q\left(1-\hat{\lambda}_{2}\right)-1} n}{n^{1-q}} b_{n}^{q}\right]^{1 / q} .
\end{aligned}
$$

Lemma 7. If the constant factor $k_{\lambda}^{1 / p}\left(\lambda-\lambda_{2}\right) k_{\lambda}^{1 / q}\left(\lambda_{1}\right)$ in (14) is the best possible, then $\lambda_{1}+\lambda_{2}=\lambda$.

Proof. If the constant factor $k_{\lambda}^{1 / p}\left(\lambda-\lambda_{2}\right) k_{\lambda}^{1 / q}\left(\lambda_{1}\right)$ in (14) is the best possible, then, by (27) and (17), the unique best possible constant factor must be $k_{\lambda}\left(\hat{\lambda}_{1}\right)\left(\in \mathrm{R}_{+}\right)$, namely,

$$
k_{\lambda}\left(\widehat{\lambda}_{1}\right)=k_{\lambda}^{1 / p}\left(\lambda-\lambda_{2}\right) k_{\lambda}^{1 / q}\left(\lambda_{1}\right) .
$$

We observe that (26) keeps the form of equality if and only if there exist constants $A$ and $B$, such that they are not all zero and (cf. [26])

$$
A u^{\lambda-\lambda_{2}-1}=B u^{\lambda_{1}-1} \text { a.e. in } \mathrm{R}_{+}=(0, \infty) .
$$

Assuming that $A \neq 0$ (otherwise, $B=A=0$ ), it follows that $u^{\lambda-\lambda_{2}-\lambda_{1}}=B / A$ a.e. in $\mathrm{R}_{+}$, and then $\lambda-\lambda_{2}-\lambda_{1}=0$, namely, $\lambda_{1}+\lambda_{2}=\lambda$.

\section{Main Results}

Theorem 8. Inequality (14) is equivalent to

$$
\begin{aligned}
& J:= {\left[\sum _ { n = 2 } ^ { \infty } \frac { \operatorname { l n } { } ^ { p ( ( \lambda - \lambda _ { 1 } ) / q + \lambda _ { 2 } / p ) - 1 } n } { n } \left(\sum_{m=2}^{\infty} k_{\lambda}(\ln m, \ln n)\right.\right.} \\
&\left.\left.\cdot a_{m}\right)^{p}\right]^{1 / p}<k_{\lambda}^{1 / p}\left(\lambda-\lambda_{2}\right) k_{\lambda}^{1 / q}\left(\lambda_{1}\right) \\
& \cdot\left\{\sum_{m=2}^{\infty} \frac{\ln ^{p\left[1-\left(\left(\lambda-\lambda_{2}\right) / p+\lambda_{1} / q\right)\right]-1} m}{m^{1-p}} a_{m}^{p}\right\}^{1 / p} .
\end{aligned}
$$

If the constant factor in (14) is the best possible, then so is the constant factor in (30).

Proof. Suppose that (30) is valid. By Hölder's inequality (cf. [26]), we find

$$
\begin{aligned}
I= & \sum_{n=2}^{\infty}\left[\frac{\ln ^{-1 / p+\left(\left(\lambda-\lambda_{1}\right) / q+\lambda_{2} / p\right)} n}{n^{1 / p}} \sum_{m=2}^{\infty} k_{\lambda}(\ln m, \ln n) a_{m}\right] \\
& \cdot\left[\frac{\ln ^{1 / p-\left(\left(\lambda-\lambda_{1}\right) / q+\lambda_{2} / p\right)} n}{n^{-1 / p}} b_{n}\right] \\
& \leq J\left\{\sum_{n=2}^{\infty} \frac{\ln q\left[1-\left(\left(\lambda-\lambda_{1}\right) / q+\lambda_{2} / p\right)\right]-1}{n^{1-q}} b_{n}^{q}\right\}^{1 / q} .
\end{aligned}
$$

Then by (30), we obtain (14).

On the other hand, assuming that (14) is valid, we set

$b_{n}$

$$
:=\frac{\ln ^{p\left(\left(\lambda-\lambda_{1}\right) / q+\lambda_{2} / p\right)-1} n}{n}\left(\sum_{m=2}^{\infty} k_{\lambda}(\ln m, \ln n) a_{m}\right)^{p-1},
$$

$$
n \in \mathbf{N} \backslash\{1\} .
$$

If $J=0$, then (30) is naturally valid; if $J=\infty$, then it is impossible to make (30) valid, namely, $J<\infty$. Suppose that $0<J<\infty$. By (14), it follows that

$$
\begin{aligned}
& \sum_{n=2}^{\infty} \frac{\ln ^{q\left[1-\left(\left(\lambda-\lambda_{1}\right) / q+\lambda_{2} / p\right)\right]-1} n}{n^{1-q}} b_{n}^{q}=J^{p}=I \\
& <k_{\lambda}^{1 / p}\left(\lambda-\lambda_{2}\right) k_{\lambda}^{1 / q}\left(\lambda_{1}\right) \\
& \cdot\left\{\sum_{m=2}^{\infty} \frac{\ln ^{p\left[1-\left(\left(\lambda-\lambda_{2}\right) / p+\lambda_{1} / q\right)\right]-1} m}{m^{1-p}} a_{m}^{p}\right\}^{1 / p} \\
& \cdot\left\{\sum_{n=2}^{\infty} \frac{\ln ^{q\left[1-\left(\left(\lambda-\lambda_{1}\right) / q+\lambda_{2} / p\right)\right]-1} n}{n^{1-q}} b_{n}^{q}\right\}^{1 / q},
\end{aligned}
$$




$$
\begin{aligned}
J & =\left\{\sum_{n=2}^{\infty} \frac{\ln ^{q\left[1-\left(\left(\lambda-\lambda_{1}\right) / q+\lambda_{2} / p\right)\right]-1} n}{n^{1-q}} b_{n}^{q}\right\}^{1 / p} \\
& <k_{\lambda}^{1 / p}\left(\lambda-\lambda_{2}\right) k_{\lambda}^{1 / q}\left(\lambda_{1}\right) \\
& \cdot\left\{\sum_{m=2}^{\infty} \frac{\ln ^{p\left[1-\left(\left(\lambda-\lambda_{2}\right) / p+\lambda_{1} / q\right)\right]-1} m}{m^{1-p}} a_{m}^{p}\right\}^{1 / p},
\end{aligned}
$$

namely, (30) follows, which is equivalent to (14).

If the constant factor in (14) is the best possible, then so is constant factor in (30). Otherwise, by (31), we would reach a contradiction that the constant factor in (14) is not the best possible.

Theorem 9. The statements (i), (ii), (iii), and (iv) are equivalent as follows:

(i) $k_{\lambda}^{1 / p}\left(\lambda-\lambda_{2}\right) k_{\lambda}^{1 / q}\left(\lambda_{1}\right)$ is independent of $p, q$

(ii) $k_{\lambda}^{1 / p}\left(\lambda-\lambda_{2}\right) k_{\lambda}^{1 / q}\left(\lambda_{1}\right)$ is expressible as a single integral

(iii) $k_{\lambda}^{1 / p}\left(\lambda-\lambda_{2}\right) k_{\lambda}^{1 / q}\left(\lambda_{1}\right)$ is the best possible constant factor of (14)

(iv) $\lambda_{1}+\lambda_{2}=\lambda$

If the statement (iv) follows, namely, $\lambda_{1}+\lambda_{2}=\lambda$, then we have (17) and the following equivalent inequality with the best possible constant factor $k_{\lambda}\left(\lambda_{1}\right)$ :

$$
\begin{gathered}
{\left[\sum_{n=2}^{\infty} \frac{\ln ^{p \lambda_{2}-1} n}{n}\left(\sum_{m=2}^{\infty} k_{\lambda}(\ln m, \ln n) a_{m}\right)^{p}\right]^{1 / p}} \\
<k_{\lambda}\left(\lambda_{1}\right)\left[\sum_{m=2}^{\infty} \frac{\ln ^{p\left(1-\lambda_{1}\right)-1} m}{m^{1-p}} a_{m}^{p}\right]^{1 / p}
\end{gathered}
$$

Proof. (i) $=>$ (ii). Since $k_{\lambda}^{1 / p}\left(\lambda-\lambda_{2}\right) k_{\lambda}^{1 / q}\left(\lambda_{1}\right)$ is independent of $p, q$, we find

$$
\begin{aligned}
k_{\lambda}^{1 / p} & \left(\lambda-\lambda_{2}\right) k_{\lambda}^{1 / q}\left(\lambda_{1}\right) \\
\quad= & \lim _{p \rightarrow \infty} \lim _{q \rightarrow 1^{+}} k_{\lambda}^{1 / p}\left(\lambda-\lambda_{2}\right) k_{\lambda}^{1 / q}\left(\lambda_{1}\right)=k_{\lambda}\left(\lambda_{1}\right),
\end{aligned}
$$

namely, $k_{\lambda}^{1 / p}\left(\lambda-\lambda_{2}\right) k_{\lambda}^{1 / q}\left(\lambda_{1}\right)$ is expressible as a single integral

$$
k_{\lambda}\left(\lambda_{1}\right)=\int_{0}^{\infty} k_{\lambda}(u, 1) u^{\lambda_{1}-1} d u
$$

(ii) $=>$ (iv). In (26), if $k_{\lambda}^{1 / p}\left(\lambda-\lambda_{2}\right) k_{\lambda}^{1 / q}\left(\lambda_{1}\right)$ is expressible as a single integral $k_{\lambda}\left(\left(\lambda-\lambda_{2}\right) / p+\lambda_{1} / q\right)$, then (26) keeps the form of equality, which follows that $\lambda_{1}+\lambda_{2}=\lambda$.

(iv) $=>$ (i). If $\lambda_{1}+\lambda_{2}=\lambda$, then $k_{\lambda}^{1 / p}\left(\lambda-\lambda_{2}\right) k_{\lambda}^{1 / q}\left(\lambda_{1}\right)=$ $k_{\lambda}\left(\lambda_{1}\right)$, which is independent of $p, q$. Hence, we have (i) $\Longleftrightarrow$ (ii) $\Longleftrightarrow$ (iv).

(iii) $=>$ (iv). By Lemma 7, we have $\lambda_{1}+\lambda_{2}=\lambda$. (iv) $=>$ (iii). By Lemma 5, for $\lambda_{1}+\lambda_{2}=\lambda, k_{\lambda}^{1 / p}(\lambda-$ $\left.\lambda_{2}\right) k_{\lambda}^{1 / q}\left(\lambda_{1}\right)\left(=k_{\lambda}\left(\lambda_{1}\right)\right)$ is the best possible constant factor of (14). Therefore, we have (iii) $\Longleftrightarrow$ (iv).

Hence, the statements (i), (ii), (iii), and (iv) are equivalent.

Remark 10. (i) For $\lambda=1, \lambda_{1}=1 / q, \lambda_{2}=1 / p$ in (17) and (34), we have the following equivalent inequalities with the best possible constant factor $k_{1}(1 / q)$ :

$$
\begin{aligned}
& \sum_{n=2}^{\infty} \sum_{m=2}^{\infty} k_{1}(\ln m, \ln n) a_{m} b_{n} \\
& \quad<k_{1}\left(\frac{1}{q}\right)\left(\sum_{m=2}^{\infty} \frac{a_{m}^{p}}{m^{1-p}}\right)^{1 / p}\left(\sum_{n=2}^{\infty} \frac{b_{n}^{q}}{n^{1-q}}\right)^{1 / q}, \\
& {\left[\sum_{n=2}^{\infty} \frac{1}{n}\left(\sum_{m=2}^{\infty} k_{1}(\ln m, \ln n) a_{m}\right)^{p}\right]^{1 / p}} \\
& \quad<k_{1}\left(\frac{1}{q}\right)\left(\sum_{m=2}^{\infty} \frac{a_{m}^{p}}{m^{1-p}}\right)^{1 / p} .
\end{aligned}
$$

(ii) For $\lambda=1, \lambda_{1}=1 / p, \lambda_{2}=1 / q$ in (17) and (34), we have the following equivalent inequalities with the best possible constant factor $k_{1}(1 / p)$ :

$$
\begin{aligned}
& \sum_{n=2}^{\infty} \sum_{m=2}^{\infty} k_{1}(\ln m, \ln n) a_{m} b_{n} \\
& <k_{1}\left(\frac{1}{p}\right)\left(\sum_{m=2}^{\infty} \frac{\ln ^{p-2} m}{m^{1-p}} a_{m}^{p}\right)^{1 / p}\left(\sum_{n=2}^{\infty} \frac{\ln ^{p-2} n}{n^{1-q}} b_{n}^{q}\right)^{1 / q}, \\
& {\left[\sum_{n=2}^{\infty} \frac{\ln ^{p-2} n}{n}\left(\sum_{m=2}^{\infty} k_{1}(\ln m, \ln n) a_{m}\right)^{p}\right]^{1 / p}} \\
& <k_{1}\left(\frac{1}{p}\right)\left(\sum_{m=2}^{\infty} \frac{\ln ^{p-2} m}{m^{1-p}} a_{m}^{p}\right)^{1 / p} .
\end{aligned}
$$

(iii) For $p=q=2$, both (37) and (39) reduce to

$$
\begin{aligned}
& \sum_{n=2}^{\infty} \sum_{m=2}^{\infty} k_{1}(\ln m, \ln n) a_{m} b_{n} \\
& \quad<k_{1}\left(\frac{1}{2}\right)\left(\sum_{m=2}^{\infty} m a_{m}^{2} \sum_{n=2}^{\infty} n b_{n}^{2}\right)^{1 / 2},
\end{aligned}
$$

and both (38) and (40) reduce to the equivalent form of (41) as follows:

$$
\begin{gathered}
{\left[\sum_{n=2}^{\infty} \frac{1}{n}\left(\sum_{m=2}^{\infty} k_{1}(\ln m, \ln n) a_{m}\right)^{2}\right]^{1 / 2}} \\
<k_{1}\left(\frac{1}{2}\right)\left(\sum_{m=2}^{\infty} m a_{m}^{2}\right)^{1 / 2} .
\end{gathered}
$$




\section{Operator Expressions and Some Particular Cases}

We set functions

$$
\begin{aligned}
& \varphi(m):=\frac{\ln ^{p\left[1-\left(\left(\lambda-\lambda_{2}\right) / p+\lambda_{1} / q\right)\right]-1} m}{m^{1-p}} \\
& \psi(n):=\frac{\ln ^{q\left[1-\left(\left(\lambda-\lambda_{1}\right) / q+\lambda_{2} / p\right)\right]-1} n}{n^{1-q}},
\end{aligned}
$$

where

$$
\psi^{1-p}(n)=\frac{\ln ^{p\left(\left(\lambda-\lambda_{1}\right) / q+\lambda_{2} / p\right)-1} n}{n} \quad(m, n \in \mathrm{N} \backslash\{1\}) .
$$

Define the following real normed spaces:

$$
\begin{aligned}
l_{p, \varphi} & :=\left\{a=\left\{a_{m}\right\}_{m=2}^{\infty} ;\|a\|_{p, \varphi}:=\left(\sum_{m=2}^{\infty} \varphi(m)\left|a_{m}\right|^{p}\right)^{1 / p}\right. \\
< & \infty\}, \\
l_{q, \psi} & :=\left\{b=\left\{b_{n}\right\}_{n=2}^{\infty} ;\|b\|_{q, \psi}:=\left(\sum_{n=2}^{\infty} \psi(n)\left|b_{n}\right|^{q}\right)^{1 / q}\right. \\
< & \infty\}, \\
l_{p, \psi^{1-p}} & :=\left\{c=\left\{c_{n}\right\}_{n=2}^{\infty} ;\|c\|_{p, \psi^{1-p}}\right. \\
& \left.:=\left(\sum_{n=2}^{\infty} \psi^{1-p}(n)\left|c_{n}\right|^{p}\right)^{1 / p}<\infty\right\} .
\end{aligned}
$$

Assuming that $a \in l_{p, \varphi}$, setting

$$
\begin{aligned}
c & =\left\{c_{n}\right\}_{n=2}^{\infty}, \\
c_{n} & :=\sum_{m=2}^{\infty} k_{\lambda}(\ln m, \ln n) a_{m},
\end{aligned}
$$

$$
n \in \mathrm{N} \backslash\{1\}
$$

we can rewrite (30) as follows:

$$
\|c\|_{p, \psi^{1-p}}<k_{\lambda}^{1 / p}\left(\lambda-\lambda_{2}\right) k_{\lambda}^{1 / q}\left(\lambda_{1}\right)\|a\|_{p, \varphi}<\infty
$$

namely, $c \in l_{p, \psi^{1-p}}$.

Definition 11. Define a Mulholland-type operator $T: l_{p, \varphi} \longrightarrow$ $l_{p, \psi^{1-p}}$ as follows: for any $a \in l_{p, \varphi}$, there exists a unique representation $c \in l_{p, \psi^{1-p}}$. Define the formal inner product of $T a$ and $b \in l_{q, \psi}$ and the norm of $T$ as follows:

$$
\begin{aligned}
(T a, b) & :=\sum_{n=2}^{\infty}\left(\sum_{m=2}^{\infty} k_{\lambda}(\ln m, \ln n) a_{m}\right) b_{n}, \\
\|T\| & :=\sup _{a(\neq \theta) \in l_{p, \varphi}} \frac{\|T a\|_{p, \psi^{1-p}}}{\|a\|_{p, \varphi}} .
\end{aligned}
$$

By Theorems 8 and 9, we have the following.
Theorem 12. If $a \in l_{p, \varphi}, b \in l_{q, \psi},\|a\|_{p, \varphi},\|b\|_{q, \psi}>0$, then we have the following equivalent inequalities:

$$
\begin{aligned}
(T a, b) & <k_{\lambda}^{1 / p}\left(\lambda-\lambda_{2}\right) k_{\lambda}^{1 / q}\left(\lambda_{1}\right)\|a\|_{p, \varphi}\|b\|_{q, \psi}, \\
\|T a\|_{p, \psi^{1-p}} & <k_{\lambda}^{1 / p}\left(\lambda-\lambda_{2}\right) k_{\lambda}^{1 / q}\left(\lambda_{1}\right)\|a\|_{p, \varphi} .
\end{aligned}
$$

Moreover, $\lambda_{1}+\lambda_{2}=\lambda$ if and only if the constant factor $k_{\lambda}^{1 / p}\left(\lambda-\lambda_{2}\right) k_{\lambda}^{1 / q}\left(\lambda_{1}\right)=k_{\lambda}\left(\lambda_{1}\right)$ in (49) and (50) is the best possible, namely,

$$
\|T\|=k_{\lambda}\left(\lambda_{1}\right)
$$

Example 13. We set $k_{\lambda}(x, y):=1 /(c x+y)^{\lambda}(c, \lambda>0 ; x, y>$ $0)$. Then we find $k_{\lambda}(\ln m, \ln n)=1 / \ln ^{\lambda} m^{c} n$. For $0<\lambda_{i}, \lambda-$ $\lambda_{i} \leq 1(i=1,2), k_{\lambda}(x, y)$ is a positive homogeneous function of degree $-\lambda$, such that $k_{\lambda}(x, y)$ is decreasing with respect to $x, y>0$, and for $\gamma=\lambda_{1}, \lambda-\lambda_{2}$,

$$
\begin{aligned}
k_{\lambda}(\gamma) & =\int_{0}^{\infty} \frac{u^{\gamma-1}}{(c u+1)^{\lambda}} d u=\frac{1}{c^{\gamma}} \int_{0}^{\infty} \frac{v^{\gamma-1}}{(v+1)^{\lambda}} d v \\
& =\frac{1}{c^{\gamma}} B(\gamma, \lambda-\gamma) \in \mathrm{R}_{+} .
\end{aligned}
$$

In view of Theorem 12, it follows that $\lambda_{1}+\lambda_{2}=\lambda$ if and only if

$$
\|T\|=k_{\lambda}\left(\lambda_{1}\right)=\frac{1}{c^{\lambda_{1}}} B\left(\lambda_{1}, \lambda_{2}\right)
$$

Example 14. We set $k_{\lambda}(x, y):=\ln (c x / y) /(c x)^{\lambda}-y^{\lambda}(c>$ $0, \lambda>0 ; x, y>0)$. Then we find $k_{\lambda}(\ln m, \ln n)=$ $\ln \left(\ln m^{c} / \ln n\right) /\left(\ln ^{\lambda} m^{c}-\ln ^{\lambda} n\right)$. For $0<\lambda_{i}, \lambda-\lambda_{i} \leq 1(i=1,2)$, $k_{\lambda}(x, y)$ is a positive homogeneous function of degree $-\lambda$, such that $k_{\lambda}(x, y)$ is decreasing with respect to $x, y>0$ (cf. [2], Example 2.2.1), and for $\gamma=\lambda_{1}, \lambda-\lambda_{2}$,

$$
\begin{aligned}
k_{\lambda}(\gamma) & =\int_{0}^{\infty} \frac{u^{\gamma-1} \ln (c u)}{(c u)^{\lambda}-1} d u \\
& =\frac{1}{c^{\gamma} \lambda^{2}} \int_{0}^{\infty} \frac{v^{(\gamma / \lambda)-1} \ln v}{v-1} d v \\
& =\frac{1}{c^{\gamma}}\left[\frac{\pi}{\lambda \sin (\pi \gamma / \lambda)}\right]^{2} \in \mathrm{R}_{+} .
\end{aligned}
$$

In view of Theorem 12, it follows that $\lambda_{1}+\lambda_{2}=\lambda$ if and only if

$$
\|T\|=k_{\lambda}\left(\lambda_{1}\right)=\frac{1}{c^{\lambda_{1}}}\left[\frac{\pi}{\lambda \sin \left(\pi \lambda_{1} / \lambda\right)}\right]^{2} .
$$

Example 15. We set $k_{\lambda}(x, y):=1 / \prod_{k=1}^{s}\left(x^{\lambda / s}+c_{k} y^{\lambda / s}\right)(0<$ $\left.c_{1} \leq \cdots \leq c_{s}, \lambda>0 ; x, y>0\right)$. Then we find

$$
k_{\lambda}(\ln m, \ln n)=\frac{1}{\prod_{k=1}^{s}\left(\ln ^{\lambda / s} m+c_{k} \ln ^{\lambda / s} n\right)} .
$$


For $0<\lambda_{i}, \lambda-\lambda_{i} \leq 1(i=1,2), k_{\lambda}(x, y)$ is a positive homogeneous function of degree $-\lambda$, such that $k_{\lambda}(x, y)$ is decreasing with respect to $x, y>0$, and for $\gamma=\lambda_{1}, \lambda-\lambda_{2}$, by Example 1 of [28], it follows that

$$
\begin{aligned}
k_{\lambda}^{(s)}(\gamma) & =\int_{0}^{\infty} \frac{t^{\gamma-1}}{\prod_{k=1}^{s}\left(t^{\lambda / s}+c_{k}\right)} d t \\
& =\frac{\pi s}{\lambda \sin (\pi s \gamma / \lambda)} \sum_{k=1}^{s} c_{k}^{s \gamma / \lambda-1} \prod_{j=1(j \neq k)}^{s} \frac{1}{c_{j}-c_{k}} \\
& \in \mathrm{R}_{+} .
\end{aligned}
$$

In view of Theorem 12, it follows that $\lambda_{1}+\lambda_{2}=\lambda$ if and only if

$$
\begin{aligned}
\|T\| & =k_{\lambda}{ }^{(s)}\left(\lambda_{1}\right) \\
& =\frac{\pi s}{\lambda \sin \left(\pi s \lambda_{1} / \lambda\right)} \sum_{k=1}^{s} c_{k}^{s \lambda_{1} / \lambda-1} \prod_{j=1(j \neq k)}^{s} \frac{1}{c_{j}-c_{k}} .
\end{aligned}
$$

In particular, for $c_{1}=\cdots=c_{s}=c$, we have $k_{\lambda}(x, y)=$ $1 /\left(x^{\lambda / s}+c y^{\lambda / s}\right)^{s}$ and

$$
\begin{aligned}
\|T\| & =\tilde{k}_{\lambda}^{(s)}\left(\lambda_{1}\right):=\int_{0}^{\infty} \frac{t^{\lambda_{1}-1}}{\left(t^{\lambda / s}+c\right)^{s}} d t \\
& =\frac{s}{\lambda c^{\left[1-\left(\lambda_{1} / \lambda\right)\right] s}} \int_{0}^{\infty} \frac{v^{s \lambda_{1} / \lambda-1}}{(v+1)^{s}} d v \\
& =\frac{s}{\lambda c^{\left[1-\left(\lambda_{1} / \lambda\right)\right] s}} B\left(\frac{s \lambda_{1}}{\lambda}, \frac{s \lambda_{2}}{\lambda}\right) .
\end{aligned}
$$

If $s=1$, then we have $k_{\lambda}(x, y)=1 /\left(x^{\lambda}+c y^{\lambda}\right), k_{\lambda}(\ln m, \ln n)=$ $1 /\left(\ln ^{\lambda} m+c \ln ^{\lambda} n\right)$, and

$$
\|T\|=\tilde{k}_{\lambda}{ }^{(1)}\left(\lambda_{1}\right)=\frac{1}{\lambda c^{1-\left(\lambda_{1} / \lambda\right)}} \frac{\pi}{\sin \left(\pi \lambda_{1} / \lambda\right)} .
$$

\section{Conclusions}

In this paper, by the use of the weight functions and the idea of introducing parameters, a discrete Mulholland-type inequality with the general homogeneous kernel and the equivalent form are given in Lemma 3 and Theorem 8 . The equivalent statements of the best possible constant factor related to a few parameters are considered in Theorem 9. As applications, the operator expressions and some particular examples are given in Theorem 12 and Examples 13-15. The lemmas and theorems provide an extensive account of this type of inequalities.

\section{Data Availability}

The study belongs to pure theory research. There are not any sharing data.

\section{Conflicts of Interest}

The authors declare that they have no competing interests.

\section{Acknowledgments}

This work is supported by the National Natural Science Foundation (Nos. 61562016 and 51765012) and Science and Technology Planning Project Item of Guangzhou City (No. 201707010229). We are grateful for this help.

\section{References}

[1] G. H. Hardy, J. E. Littlewood, and G. Polya, Inequalities, Cambridge University Press, 1934.

[2] B. C. Yang, The Norm of Operator and Hilbert-Type Inequalities, Science Press, Beijing, China, 2009.

[3] B. C. Yang, Hilbert-Type Integral Inequalities, Bentham Science Publishers, The United Arab Emirates, 2009.

[4] M. Krnić and J. Pečarić, "General hilbert's and hardy's inequalities," Mathematical Inequalities \& Applications, vol. 8, no. 1, pp. 29-51, 2005.

[5] I. Perić and P. Vuković, "Multiple Hilbert's type inequalities with a homogeneous kernel," Banach Journal of Mathematical Analysis, vol. 5, no. 2, pp. 33-43, 2011.

[6] Q. L. Huang, "A new extension of Hardy-Hilbert-type inequality," Journal of Inequalities and Applications, vol. 2015, article 397, 12 pages, 2015.

[7] B. He and Q. Wang, "A multiple Hilbert-type discrete inequality with a new kernel and best possible constant factor," Journal of Mathematical Analysis and Applications, vol. 431, no. 2, pp. 889902, 2015.

[8] J. S. Xu, "Hardy-Hilbert's inequalities with two parameters," Advances in Mathematics, vol. 36, no. 2, pp. 63-76, 2007.

[9] Z. T. Xie, Z. Zeng, and Y. F. Sun, "A new Hilbert-type inequality with the homogeneous kernel of degree -2," Advances and Applications in Mathematical Sciences, vol. 12, no. 7, pp. 391-401, 2013.

[10] Z. Zhen, R. R. K. Gandhi, and Z. T. Xie, "A new Hilbert-type inequality with the homogeneous kernel of degree -2 and with the integral," Bulletin of Mathematical Sciences and Applications, vol. 3, no. 1, pp. 11-20, 2014.

[11] D. M. Xin, "A Hilbert-type integral inequality with the homogeneous kernel of zero degree," Mathematical Theory and Applications, vol. 30, no. 2, pp. 70-74, 2010.

[12] L. E. Azar, "The connection between Hilbert and HARdy inequalities," Journal of Inequalities and Applications, vol. 2013, article 452, 10 pages, 2013.

[13] V. Adiyasuren, T. Batbold, and M. Krnić, "Hilbert-type inequalities involving differential operators, the best constants, and applications," Mathematical Inequalities \& Applications, vol. 18, no. 1, pp. 111-124, 2015.

[14] M. T. Rassias and B. Yang, "On half-discrete Hilbert's inequality," Applied Mathematics and Computation, vol. 220, pp. 75-93, 2013.

[15] B. Yang and M. Krnić, "A half-discrete Hilbert-type inequality with a general homogeneous kernel of degree 0," Journal of Mathematical Inequalities, vol. 6, no. 3, pp. 401-417, 2012.

[16] M. T. Rassias and B. Yang, "A multidimensional half-discrete Hilbert-type inequality and the Riemann zeta function," Applied Mathematics and Computation, vol. 225, pp. 263-277, 2013.

[17] M. T. H. Rassias and B. C. Yang, "On a multidimensional half-discrete Hilbert - type inequality related to the hyperbolic cotangent function," Applied Mathematics and Computation, vol. 242, pp. 800-813, 2013. 
[18] Z. Huang and B. Yang, "On a half-discrete Hilbert-type inequality similar to Mulholland's inequality," Journal of Inequalities and Applications, vol. 2013, article 290, 8 pages, 2013.

[19] B. C. Yang and L. Debnath, Half-Discrete Hilbert-Type Inequalities, World Scientific Publishing, Singapore, 2014.

[20] Y. Hong and Y. Wen, "A necessary and sufficient condition of that Hilbert type series inequality with homogeneous kernel has the best constant factor," Annals Mathematica, vol. 37A, no. 3, pp. 329-336, 2016.

[21] Y. Hong, "On the structure character of Hilbert's type integral inequality with homogeneous kernel and applications," Journal of Jilin University (Science Edition), vol. 55, no. 2, pp. 189-194, 2017.

[22] Y. Hong, Q. Huang, B. Yang, and J. Liao, "The necessary and sufficient conditions for the existence of a kind of Hilbert-type multiple integral inequality with the non-homogeneous kernel and its applications," Journal of Inequalities and Applications, vol. 2017, article 316, 12 pages, 2017.

[23] D. Xin, B. Yang, and A. Wang, "Equivalent property of a Hilberttype integral inequality related to the beta function in the whole plane," Journal of Function Spaces, vol. 2018, Article ID 2691816, 8 pages, 2018.

[24] Y. Hong, B. He, and B. C. Yang, "Necessary and sufficient conditions for the validity of Hilbert type integral inequalities with a class of quasi-homogeneous kernels and its application in operator theory," Journal of Mathematical Inequalities, vol. 12, no. 3, pp. 777-788, 2018.

[25] Z. X. Huang and B. C. Yang, "Equivalent property of a half-discrete Hilbert's inequality with parameters," Journal of Inequalities and Applications, vol. 2018, article 333, 11 pages, 2018.

[26] J. C. Kuang, Applied Inequalities, Shangdong Science and Technology Press, Jinan, China, 2004.

[27] J. C. Kuang, Real Analysis and Functional Analysis (Continuation), vol. 2, Higher Education Press, Beijing, China, 2015.

[28] B. Yang, "On a more accurate multidimensional Hilbert-type inequality with parameters," Mathematical Inequalities \& Applications, vol. 18, no. 2, pp. 429-441, 2015. 


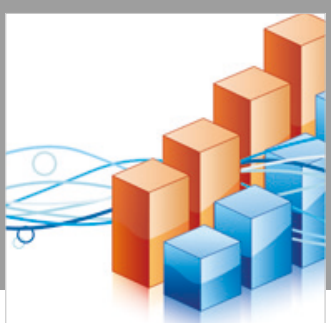

Advances in

Operations Research

\section{-n-m}
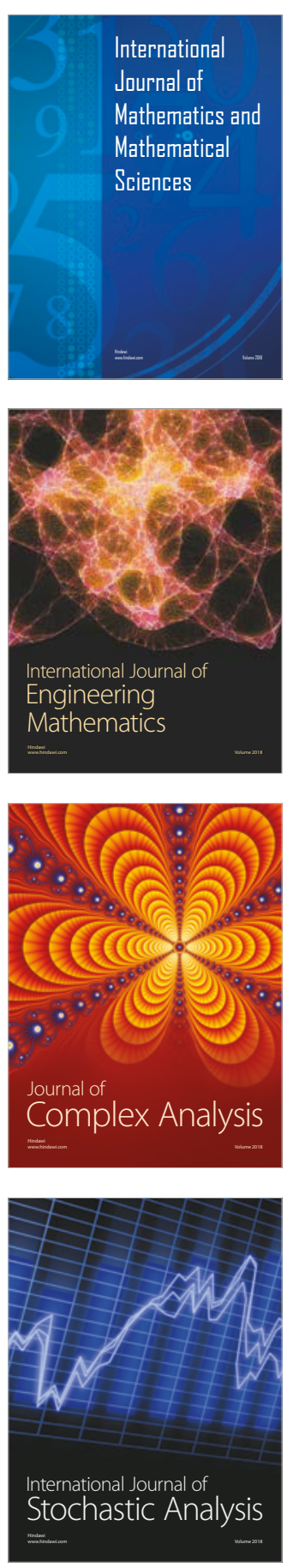
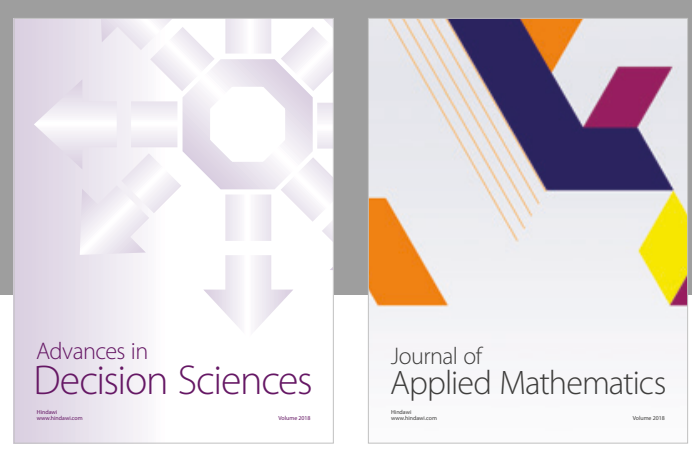

Journal of

Applied Mathematics
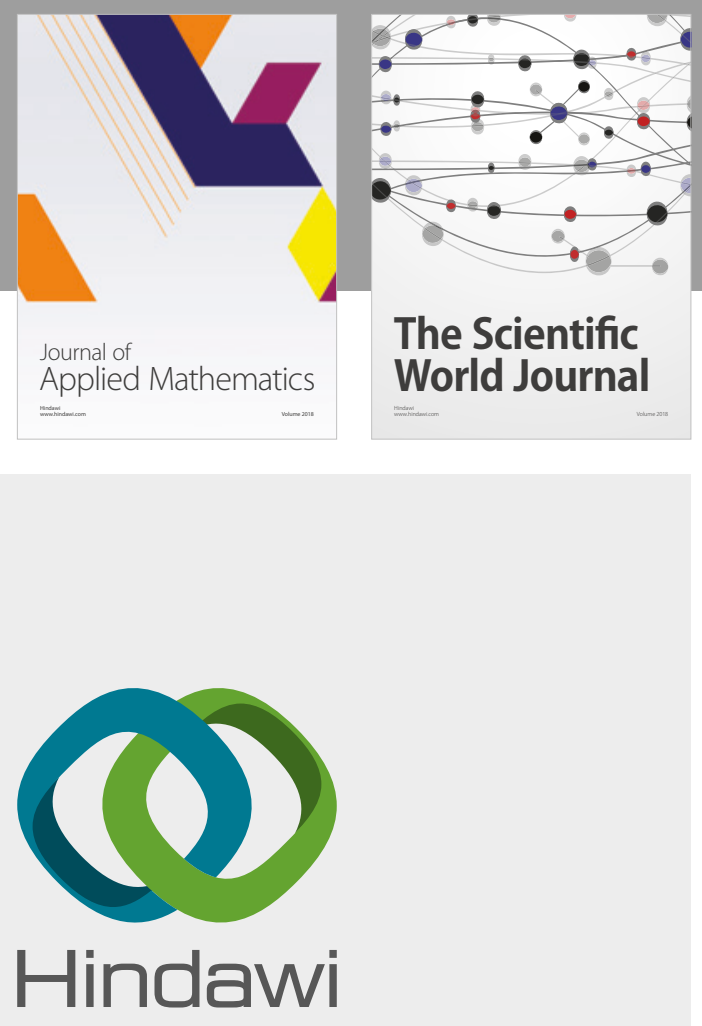

Submit your manuscripts at

www.hindawi.com

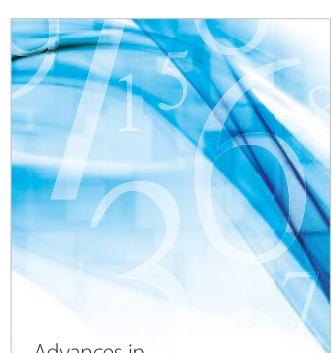

Advances in
Numerical Analysis
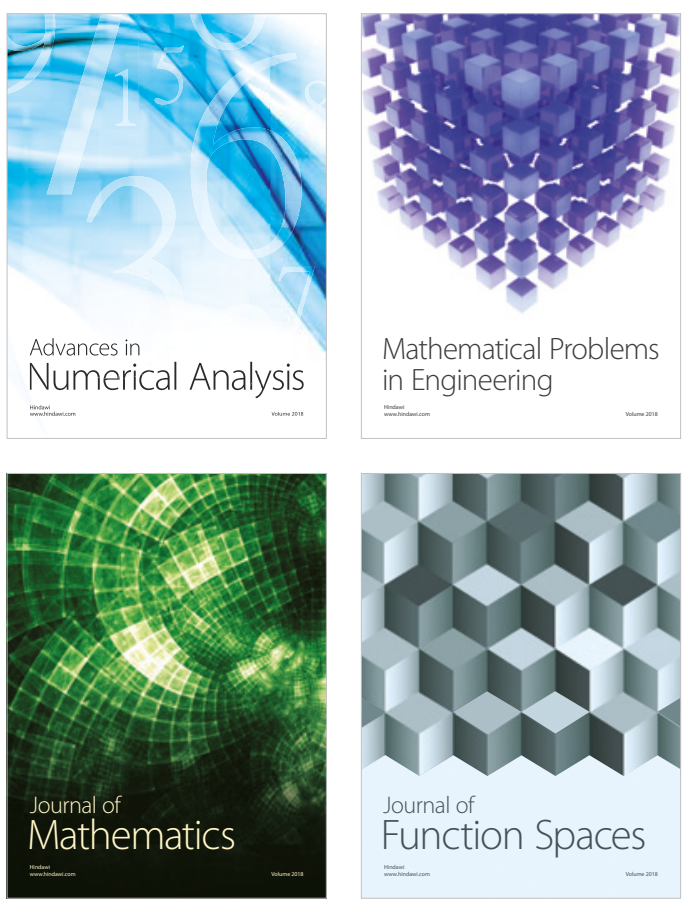

Mathematical Problems in Engineering

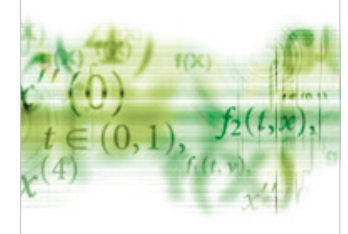

International Journal of

Differential Equations

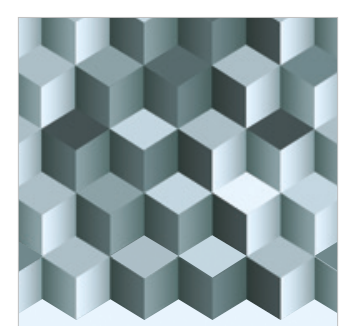

Journal of

Function Spaces

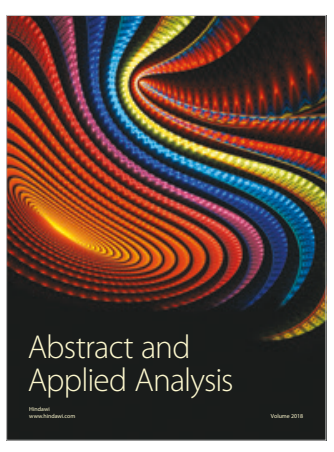

The Scientific

World Journal

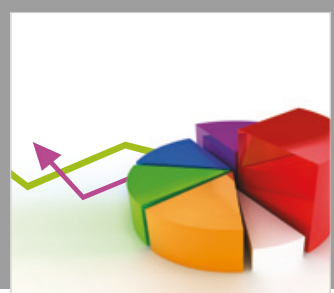

Journal of

Probability and Statistics
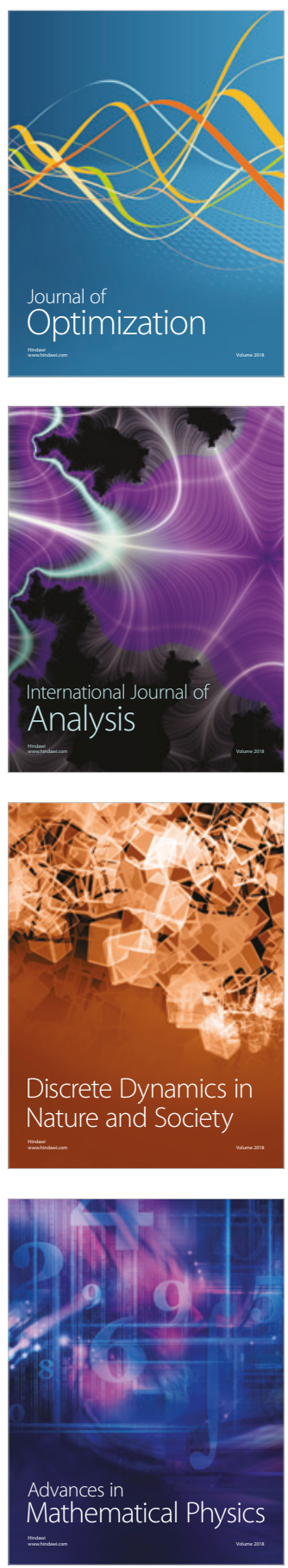